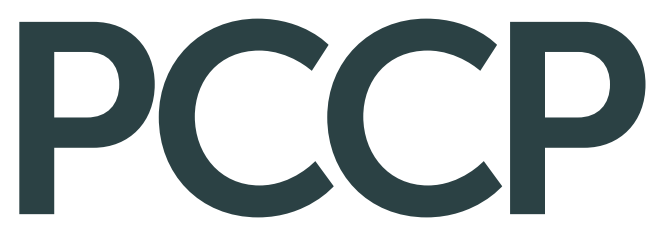

Accepted Manuscript

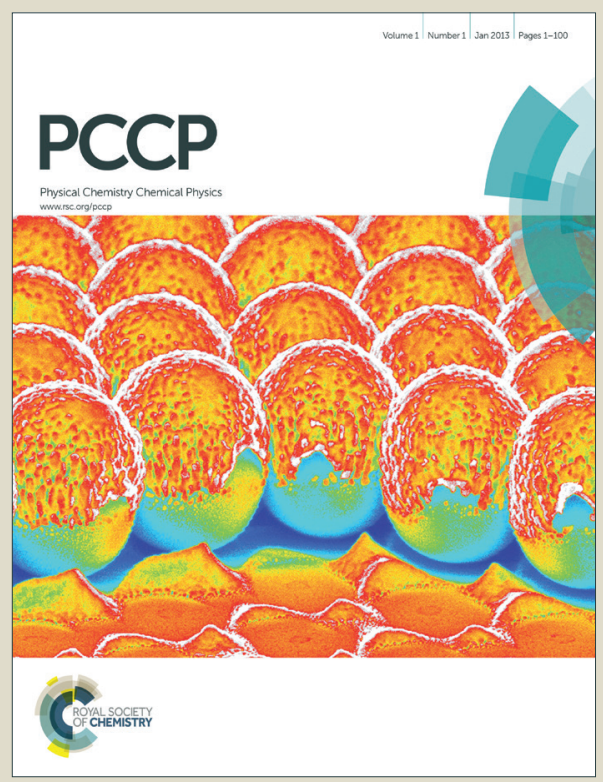

This is an Accepted Manuscript, which has been through the Royal Society of Chemistry peer review process and has been accepted for publication.

Accepted Manuscripts are published online shortly after acceptance, before technical editing, formatting and proof reading. Using this free service, authors can make their results available to the community, in citable form, before we publish the edited article. We will replace this Accepted Manuscript with the edited and formatted Advance Article as soon as it is available.

You can find more information about Accepted Manuscripts in the Information for Authors.

Please note that technical editing may introduce minor changes to the text and/or graphics, which may alter content. The journal's standard Terms \& Conditions and the Ethical guidelines still apply. In no event shall the Royal Society of Chemistry be held responsible for any errors or omissions in this Accepted Manuscript or any consequences arising from the use of any information it contains. 


\title{
Ultra-small rhenium clusters supported on graphene
}

Orlando Miramontes ${ }^{1}$, Franco Bonafé ${ }^{2}$, Ulises Santiago ${ }^{1}$, Eduardo Larios-Rodriguez ${ }^{3}$, Jesús J. Velázquez-Salazar ${ }^{1}$, Marcelo M. Mariscal ${ }^{2, *}$ and Miguel José Yacaman ${ }^{1, *}$

${ }^{1}$ Department of Physics and Astronomy, University of Texas at San Antonio

2 INFIQC, CONICET. Departamento de Matemática y Física, Facultad de Ciencias Químicas, Universidad Nacional de Córdoba Argentina.

${ }^{3}$ Departamento de Ingeniería Química y Metalurgia, Universidad de Sonora, Rosales y Luis Encinas S/N, Hermosillo, Sonora, C.P. 83000 México.

*e-mail: marcelo.mariscal@conicet.gov.ar / miguel.yacaman@utsa.edu

\begin{abstract}
The adsorption of very small rhenium clusters (2 - 13 atoms) supported on graphene was studied with high annular dark field - scanning transmission electron microscopy (HAADF-STEM). The atomic structure of the clusters was fully resolved with the aid of density functional calculations and STEM simulations. It was found that octahedral and tetrahedral structures work as seeds to obtain more complex morphologies. Finally, a detailed analysis of the electronic structure suggested that a higher catalytic effect can be expected in Re clusters when adsorbed on graphene than in isolated ones.
\end{abstract}

\section{Introduction}

Most industrial catalysts consist of expensive transition or noble-metals dispersed on inexpensive high-area porous supports. Generally, supported-metal catalysts are dispersed in nano-sized clusters to enhance the efficient use of catalytically active atoms. When the cluster size is very small, the majority of the atoms are at the surface, accessible to reactants and available for catalysis, (1) since low-coordination or unsaturated atoms often function as active sites, the few atoms clusters and single atoms are highly desirable for catalytic reactions. (2) Since the surface free energy of metals increases with decreasing particle size, coalescence of small clusters is promoted, however, the use of an appropriate scaffold material which interacts 
strongly with the metal species prevents the coalescence of individual atoms and clusters. In the limit of the scale, the material is $100 \%$ dispersed.

These clusters had been scarcely studied due to the difficulty of identifying these species by the conventional microscopic and spectroscopic techniques. Aberration corrected scanning transmission electron microscopy (STEM), particularly, High-Angle Annular Dark Field (HAADF)-STEM is a unique tool able to reveal this nanostructures, even single atoms (3-6), allowing to determine the atomic arrangement of clusters with few atoms. This is due to aberration-corrected HAADF-STEM is known for its chemical sensitivity as well as its high spatial resolution. Gold atoms and gold clusters have been identified with this technique. (7-9) However, to the best of our knowledge, experimental studies of the synthesis and structural characterization of rhenium clusters have not been reported yet.

It is known that rhenium-based materials are widely used as catalysts in petrochemical industries. With rhenium clusters the catalytic reactions in which rhenium participates could be improved greatly; also by characterizing the structure that a rhenium low-atomicity cluster forms could contribute to know how the clusters react with other compounds in chemical reactions.

In the present work we report for the first time, the synthesis of rhenium clusters and the subsequent adsorption on graphene. The ultra-small Re clusters obtained were later characterized systematically with HAADF-STEM and the intensity profiles technique, where the atom position can be solved with sub-angstrom precision. Ab-initio calculations based on density functional theory were performed to understand the atomic structures observed experimentally, as well as to predict some new properties of the new hybrid materials. Finally an inspection on the electronic structure of the Re clusters supported on graphene compared to those in vacuum show some prominent properties that should be useful to prepare ultra-small catalyst based on Re.

\section{Methods}

\section{Experimental}

Rhenium clusters were synthesized by a modified Brust method first proposed by Brust, M. et al. (10). Clusters were synthetized using a Water-Toluene system. Re+ was transferred from aqueous solution to toluene using tetraoctylammonium bromide as the phase-transfer reagent and reduced with aqueous sodium borohydride in the presence of 1-dodecanethiol $\left(\mathrm{C}_{12} \mathrm{H}_{25} \mathrm{SH}\right)$. 
A solution of tetraoctylammonium bromide $(98 \%)$ in Toluene [10mL, $0.05 \mathrm{~mol}]$ was stirred uniformly for $15 \mathrm{~min}$ and then an aqueous solution of rhenium (III) chloride [2mL, $0.05 \mathrm{~mol}$ ] was added. The solution was left stirring for 1 hour, then $0.3 \mathrm{~mL}$ of 1 -dodecanethiol was added and it was left stirring again for 1 hour. Then an aqueous substance of Sodium borohydride [6.25 mL, $0.028 \mathrm{~mol}$ ] was added slowly to the mixture and left for $40 \mathrm{~min}-1$ hour stirring. The two phases can be seen clearly, the upper phase is almost black and the lower part is light white-transparent. The upper black part is removed and washed with ethanol several times (6 - 10) in a Rotary Evaporator and re-dispersed in toluene. Since the synthesis itself requires organic compounds it was not possible to wash the clusters perfectly just with the rotary evaporator, once the solution of particles in toluene was on the grid it was necessary to wash the grid in a solution one-to-one of methanol with chloroform. Every material used for this synthesis was bought in Sigma-Aldrich.

Rhenium clusters were supported in a grid with a graphene-sheet to be characterized by aberration corrected scanning transmission electron microscopy (STEM).

\section{Aberration corrected imaging /STEM.}

Graphene-supported Re clusters were characterized by a Cs-corrected JEOL JEM-ARM 200F $200 \mathrm{kV}$ microscope equipped with a CEOS Cs corrector on the illumination system. The probe size used for acquiring the HAADF-STEM images was $<1 \AA$ (8C) with a probe current of $35 \mathrm{pA}$. The $\mathrm{Cl}$ aperture size used was $30 \mu \mathrm{m}$ and the camera length $12 \mathrm{~cm}$, corresponding to a collection angle of 33-125 mrad.

\section{Theoretical calculations}

Rhenium clusters structures in vacuum and supported on graphene were studied using molecular dynamics (MD) and density functional theory (DFT) calculations.

Approximate cluster geometries were optimized in vacuum using MD as implemented in the LAMMPS package (11). The modified embedded atom method (MEAM) potential was used to describe Re-Re interatomic forces (12). The force tolerance used was $1 \times 10^{-8} \mathrm{eV} / \AA$.

Optimized cluster geometries were then relaxed on a graphene surface within the DFT framework under the generalized gradient approximation (GGA) proposed by Perdew and Wang (PW91) (13), implemented in the Quantum ESPRESSO package (14). A plane wave basis set 
with a kinetic energy cutoff of $435 \mathrm{eV}$ and a $2 \times 2 \times 1$ uniform k-point grid were used and checked for convergence. Periodic boundary conditions and spin polarization were implemented in all calculations. The core electrons were described with ultrasoft pseudopotencials (US-PP). A variable cell relaxation of a graphene unit cell was performed to determine the equilibrium C-C distance $(1.424 \AA)$. Two graphene supercells were used: a $24 \mathrm{C}$-atom $(3 \times 2)$ rectangular supercell $(7.4 \AA \times 8.5 \AA)$ to study the interaction with a single Re atom, and a bigger $60 \mathrm{C}$-atom $(5 \times 3)$ rectangular supercell $(12.3 \AA \times 12.8 \AA)$ to study the adsorption of Re clusters. Full relaxations using the BFGS method were performed in which the energy and force convergence criteria were set to $0.0014 \mathrm{eV}$ and $0.051 \mathrm{eV} / \AA$ respectively. Bader charges were calculated using the Bader Charge Analysis code (15).

\section{Results and Discussion}

Rhenium clusters were found to be very disperse thanks to the stabilization provided from the graphene sheet (11), which made easier to see and characterize. The HAADF-STEM images (Figure 1) clearly show single Re atoms and small clusters on the graphene sheet. The diameter of each $\operatorname{Re}$ atom is $1.5 \pm 0.1 \AA$, including the blurring effects arising from the remaining higher-order aberrations and defocus. These representative images obtained at different regions of the TEM-Grid, show clearly the number of atoms in each small cluster. The sub-angstrom-resolution HAADF images in Fig. 1 reveal noticeably that Re atoms are forming low-atomicity clusters and even some of them are single atom.
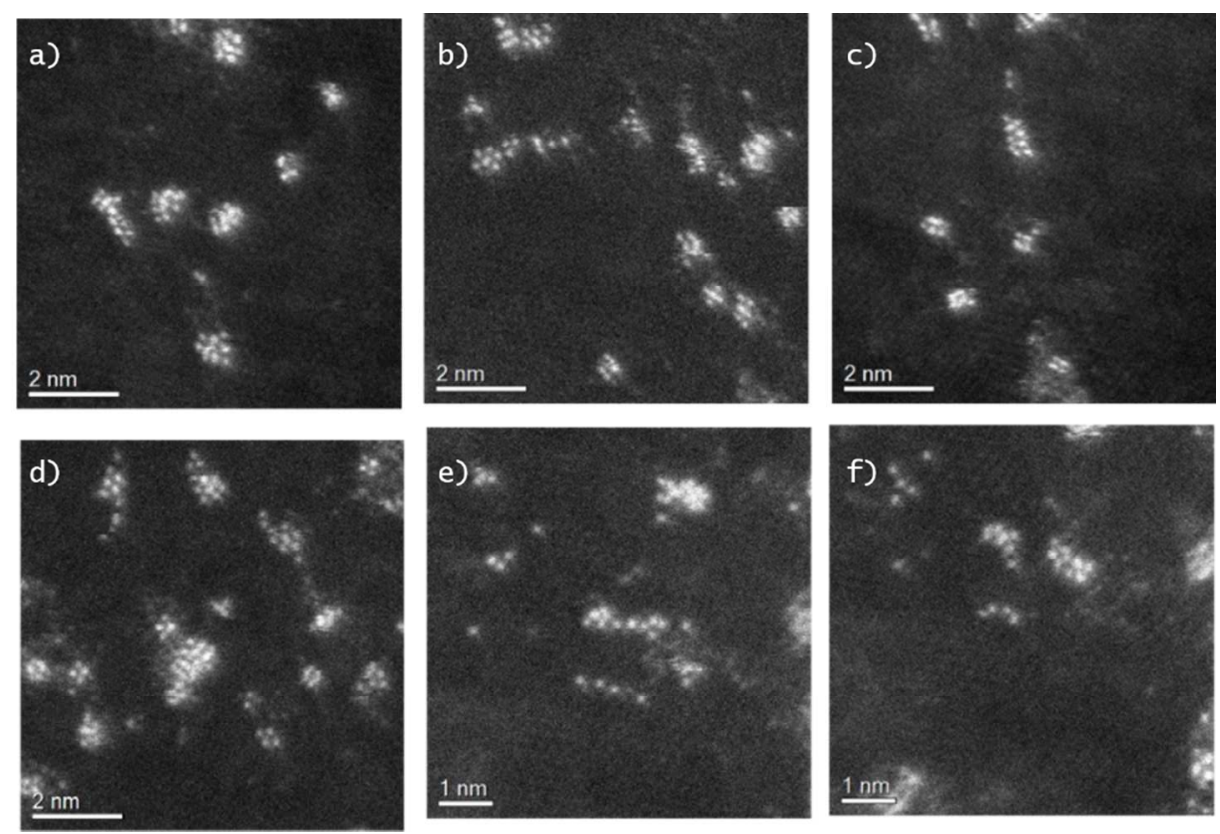
Figure 1. HAADF-STEM images of rhenium clusters of few atoms and single Re atoms.

Figure 2 shows the different intensities between spots corresponding to single or column of atoms in a low-atomicity cluster. A column with 2 rhenium atoms, spots 1 and 2 (Fig. 2b), has a $60 \%$ higher strength with respect to a single atom (Fig. $2 \mathrm{c}$ and d), spots 3 and 4 . This allows us to determine the number of atoms in each small cluster. In this cluster (Fig. 2a) we count 6 atoms. While the cluster in Figure 3 shows 8 atoms, since the intensity of the four spots is similar to each other and as high as the two-atom column in structure of the Figure 2. Thanks to this method we can figure out what's the 3D structure of the cluster. The cluster in the Figure 2 has an octahedral structure with the Re atoms in the vertexes. Applying the same method other different structures were identified.
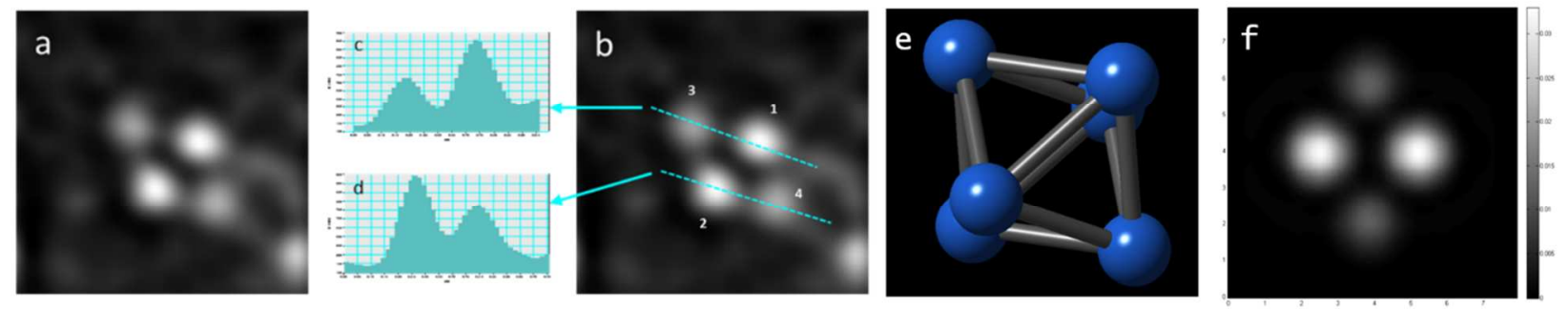

Figure 2. (a) HAADF-STEM image shows a small cluster (6 atoms), (b, c and d) the intensity profile shows different intensities for two spots in the cluster. Spots 1 and 2 show an intensity $60 \%$ greater than spots 3 and 4, (e) 3D model of the cluster, (f) STEM simulation of the proposed model.
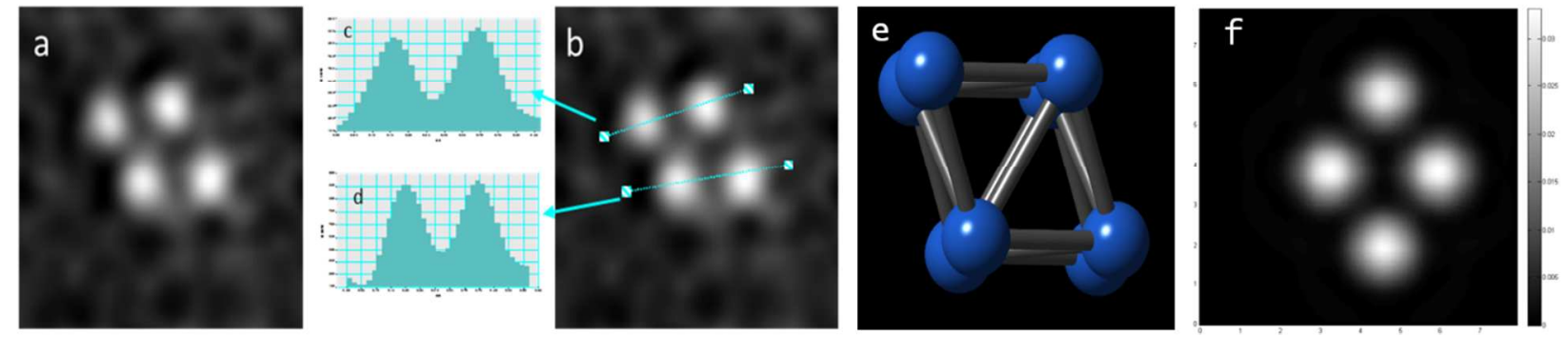

Figure 3. (a) HAADF-STEM image shows a small cluster (4 spots), (b, c and d) the intensity profile shows the same high intensity for all atoms in the cluster, (e) 3D model of the structure 
proposed, (f) STEM simulation of the structure proposed that match almost perfectly the experimental .

Even though the structures in the Figures 2 and 3 have the same projection in the 2D plane the intensities of the spots that represent the atoms are different. Following the numbering in Figure $2 \mathrm{~b}$ and applying it to the Figure $3 \mathrm{~b}$ we can tell that the spots 1 and 2 of both images have the same intensities, but the spots 3 and 4 of the Figure $3 b$ are more intense than the ones in the Figure $2 b$ and equally intense than the sports 1 and 2 of both Figures. Thanks to that analysis it is possible to know that the 3D structures of the clusters in these figures are different. However, both structures have a similar octahedral form.

Several morphologies can be observed in the structures formed by the rhenium atoms, even though every cluster is formed by a very small amount of atoms as shown in the figure 4, which shows images of (a) single atom, (c) two-atoms, (e) three-atoms, (g) four-atoms, (i) five-atoms, (k) six-atoms, (m) seven-atoms, and (o) twelve-atoms, respectively. Thus, by HAADF-STEM it is possible to determine with great accuracy the number of atoms in the cluster.

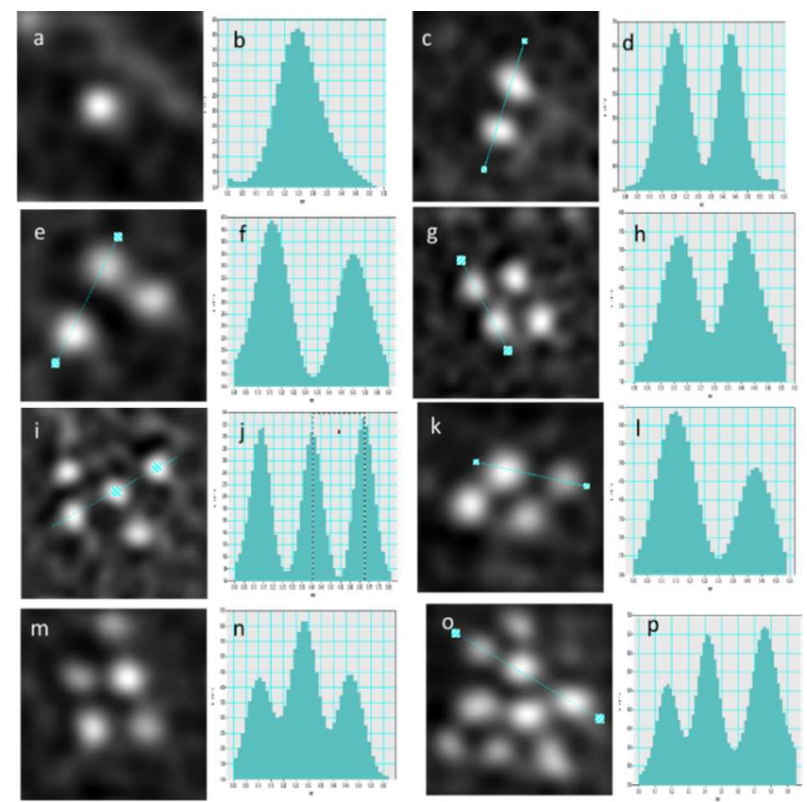

Figure 4. HAADF-STEM images for Re clusters (a) single atom, (c) 2 atoms, (e) 3 atoms, (g) 4 atoms, (i) 5 atoms, (k) 6 atoms, (m) 7 atoms, and (o) 12 atoms, respectively. 

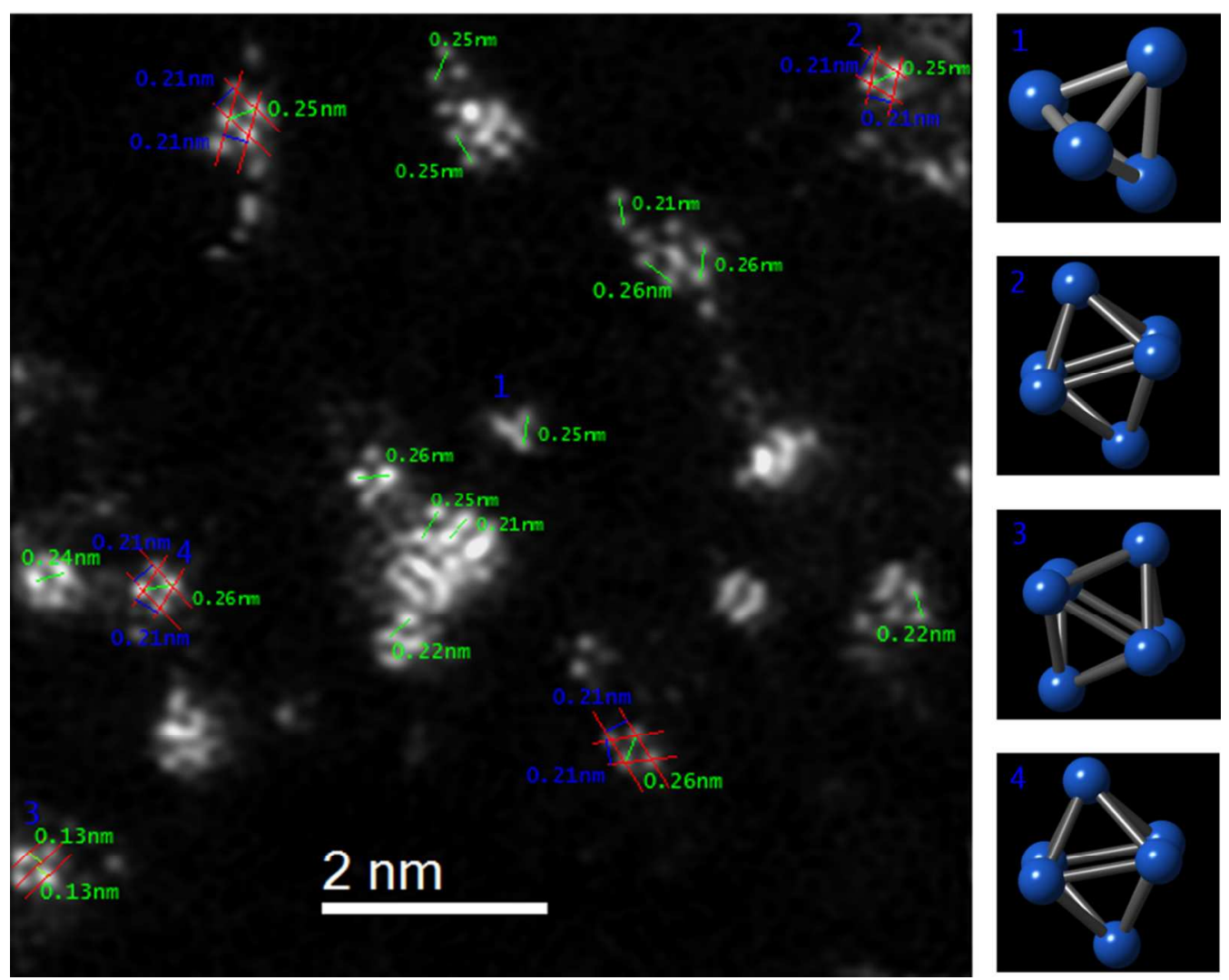

Figure 5. HAADF-STEM image with several measurements of interatomic distances between neighbor atoms (green) and between planes formed by the atoms in the structures (blue) in the clusters and the corresponding 3D models. (1) Tetrahedron (4 atoms) and (2 to 4 ) octahedra (6 atoms) in different orientations.

Various clusters were found to have very simple structures composed of a few atoms, like the ones shown in the Figure 5. However, due that the synthesis was made with 1-dodecanethiol ligands it is natural to consider that some sulfur atoms would be in the structure of the clusters and also surrounding the cluster. Nevertheless we can state that there are not sulfur atoms in the interior of the clusters because the distances between neighbors atoms in the structures do not exceed the Re-Re distance expected for the hcp structure. The distances measured in the images are just a projection in a plane of the real distance in a $3 \mathrm{D}$ structure, this is the reason why smaller distances than the bonding distance are measured, though there are not any distances 
measured greater than the distance of the Re-Re bonding, $2.74 \AA$. Also the minimum distance between Re-Re in a $\operatorname{ReS}_{2}$ structure is $2.82 \AA$, which is too big for the clusters measured.

\begin{tabular}{|l|l|l|l|l|l|}
\hline $\begin{array}{l}\text { d- } \\
\text { spacing }\end{array}$ & I(f) & h & $\mathbf{k}$ & $\mathbf{l}$ & 2-Theta \\
\hline 2.229 & 26.4 & 0 & 0 & 2 & 40.433 \\
\hline 2.106 & 100 & 1 & 0 & 1 & 42.897 \\
\hline 1.38 & 14.2 & 1 & 1 & 0 & 67.859 \\
\hline 1.262 & 14.7 & 1 & 0 & 3 & 75.232 \\
\hline
\end{tabular}

Table 1. XRD data of Rhenium (PDF \#65-3361) of interplanar distances that match the ones measured experimentally in Figure 5.

After analyzing several clusters we can state that the octahedral and tetrahedral structures work as seeds for more complex structures that were also found in the HAADF-STEM images, an example is the case of the Figures 2 and 3, in which the first structure (Figure 2) is a octahedron and the second structure (Figure 3 ) is a result of the coalescence of two tetrahedrons in which a part of the structure forms an octahedron. These structures that work as 'seeds' are very common, as we can see in the Figures 5 and 6. In Figure 6 some of the cluster seeds are compared with STEM simulations in which we can see different viewing angles of the structures.
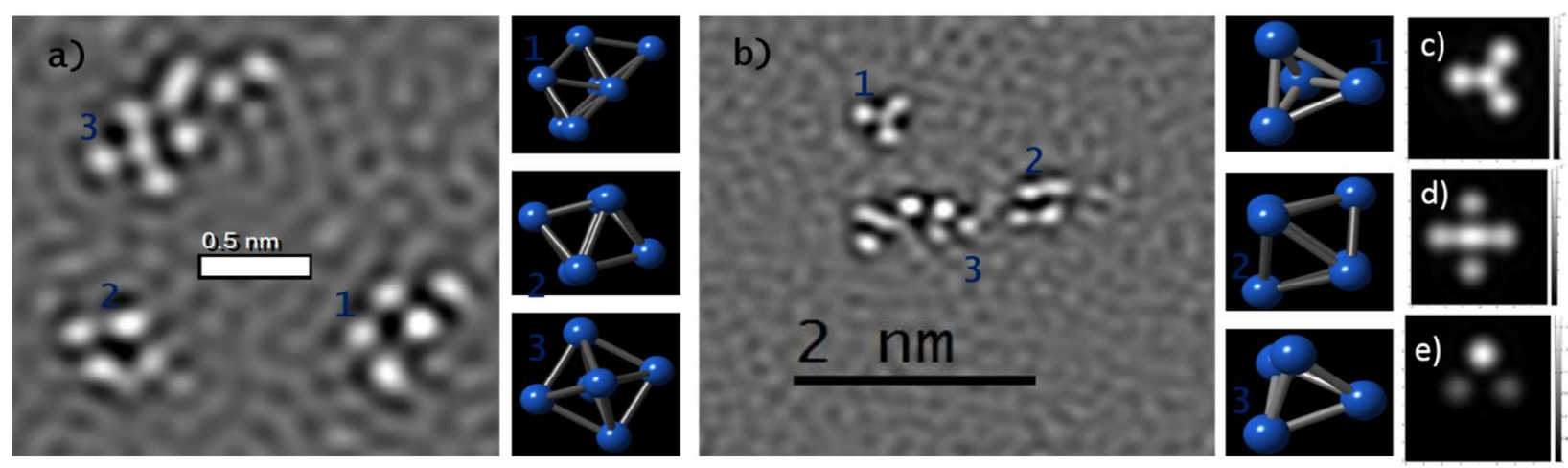

Figure 6. HAADF-STEM images. a) Two octahedral structures identified in different orientations (2 and 3 ) and one octahedral-based structure that has 2 extra atoms (1). b) 3 
identified seeds, one octahedral (2) and two tetrahedral (1 and 3), c) STEM simulation of the structure b.1, d) STEM simulation of the structure a.3, e) STEM simulation of the structure b.3.

Based in these two seeds the atomic network can be expanded in a way that the new atoms of the structure form tetrahedrons with the atoms that already are in the structure. Having this in account there can be identified more structures with a larger amount of atoms. In Figure 7 some structures are shown that are a result of the expansion of the seeds with their respective STEM simulations.
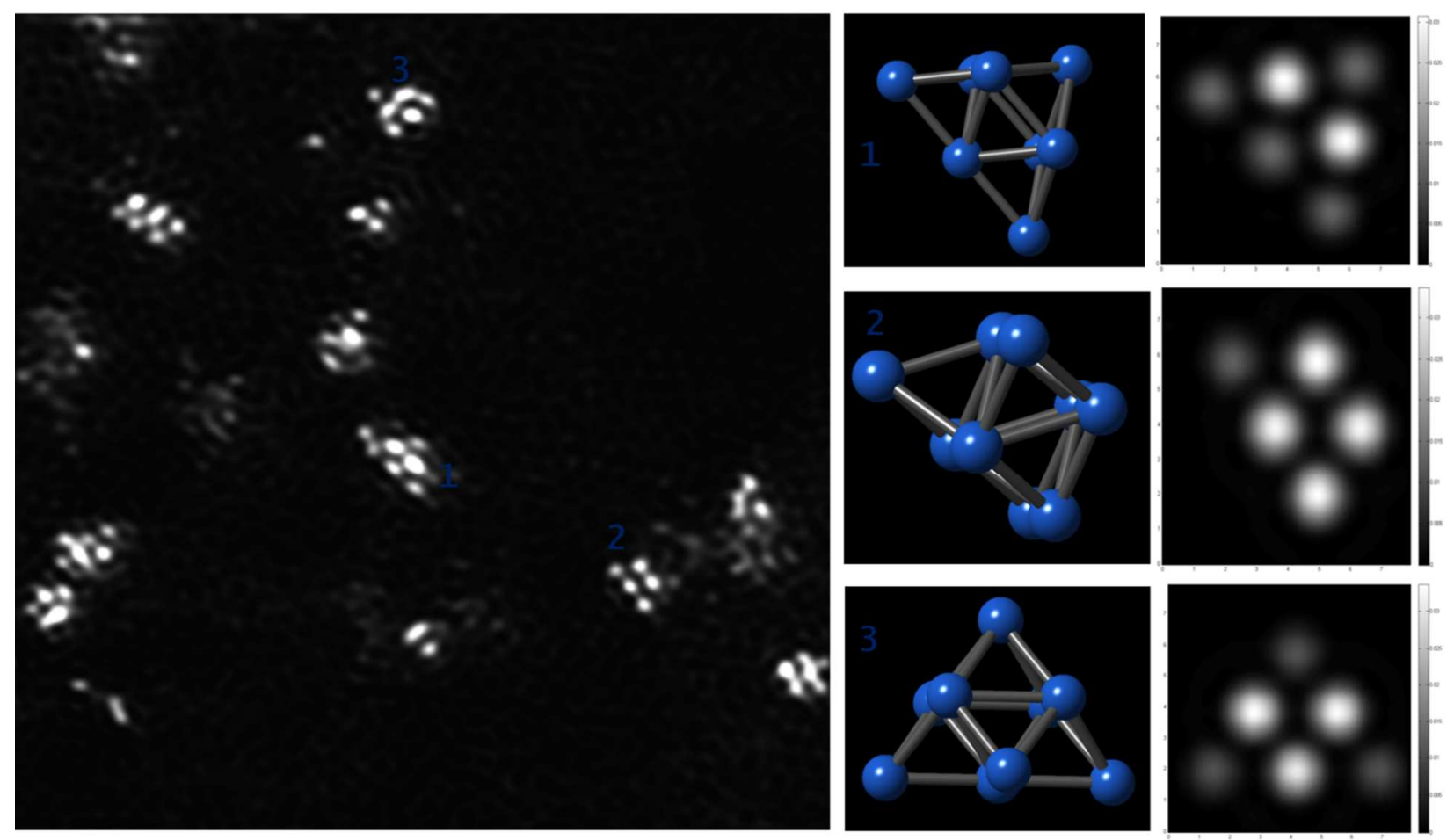

Figure 7. HAADF-STEM image showing three complex cluster structures based in the seeds planted before, octahedral and tetrahedral and their respective STEM simulation.

Calculations within the density functional theory (DFT) formalism were done in order to explain the experimental observations. The stability of the structures is defined by the adsorption energy $E_{\text {ads }}$, which was calculated as

$E_{\text {ads }}=E_{\text {Re on graphene }}-E_{\text {Re in vacuum }}-E_{\text {graphene }}$. 
Here $E_{\mathrm{Re}}$ in vacuum and $E_{\text {graphene }}$ refer to the energy of these systems optimized in the vacuum. The adsorption energy accounts for the interaction with the surface (negative term for an exothermic process) as well as the energy that arises due to the structural distortion of graphene and Re clusters when adsorbed (positive term). It is useful to consider another definition of the change in energy, called the interaction energy $E_{\text {int, }}$, which doesn't include the energy of deformation of graphene and Re clusters, and is calculated in the same way as $E_{\text {ads, }}$, but using the energy of Re clusters and graphene in the same geometry they have in the Re/graphene optimized structure. These two definitions allow us to compare not only how stabilized a cluster is when adsorbed, but also the energy needed to distort the geometries from the initial, most stable structures.

A single Re atom (adatom) was optimized in three different sites: on top of a C atom (T site), on the midpoint of a C-C bond (B site) and on a hollow position ( $\mathrm{H}$ site). It was found that the $\mathrm{H}$ site was the only stable site $\left(E_{\mathrm{ads}}=-0.136 \mathrm{eV}\right)$ while the adsorption in B or T doesn't occur; in fact, in these sites, $E_{\text {ads }} \approx 0.04 \mathrm{eV}$, which is only twice the thermal energy at $25^{\circ} \mathrm{C}$, and the adsorption distance from the surface would be about $4 \AA$. This was found by fixing the atomic position at certain distances from the surface and calculating the energy; when a relaxation was attempted on the $\mathrm{T}$ or $\mathrm{B}$ sites, the $\mathrm{Re}$ adatom relaxed into the $\mathrm{H}$ site.

The energy profile (energy vs. path coordinate) for the diffusion via high symmetry sites (hollow-bridge-hollow (H-B-H) and hollow-top-hollow (H-T-H) paths) was calculated, along with the total magnetization and the distance from the surface (see detailed description in the Supporting Information). The approximate activation energies for the H-B-H and H-T-H paths are $0.67 \mathrm{eV}$ and $0.66 \mathrm{eV}$, respectively. It is remarkable that in the spin polarized case, the energy maxima along the paths are not linked to a low stability in the middle point of the paths as could be expected, but to a change of spin caused by breaking and forming bonds. Although these energy barriers can be considered low and cause the clusters to coalesce and form bigger particles given the large cohesive energy of rhenium $(-8.03 \mathrm{eV} /$ atom) $(17)$, it is safe to say that the activation energy for cluster diffusion must be larger, since more atoms need to break and form bonds. Otherwise clusters of a few Re atoms $(<10)$ could not be observed in the experimental images. 
The properties of the adatom system were studied to explain the strong adsorption. Figure 8 (a) shows the Bader charge on each atom, represented by a color scale, and it shows a strong polarization of the system in the $z$-axis. The Re atom bears a positive charge of $+0.76 \mathrm{e}$, while the negative charge is localized mainly in the neighboring $\mathrm{C}$ atoms. The strong charge transfer is typical of bonds with ionic character, as opposed to the usual case of transition metals on graphene (18). However, charge density difference plots (Figure 8 (b) and (c)) show a considerable localization of charge in the Re-C inter-nuclear axis, regarding only the closest $\mathrm{C}$ atoms, which indicates covalent bonding. Also, the $\mathrm{C}-\mathrm{C}$ bond is weakened, as evidenced in the $0.01 \mathrm{e} / \mathrm{bohr}^{3}$ iso-surface in Figure 8 (c). Further evidence for the mixed ionic-covalent bonding is explained in the Supporting Information by analyzing the total and projected density of states.
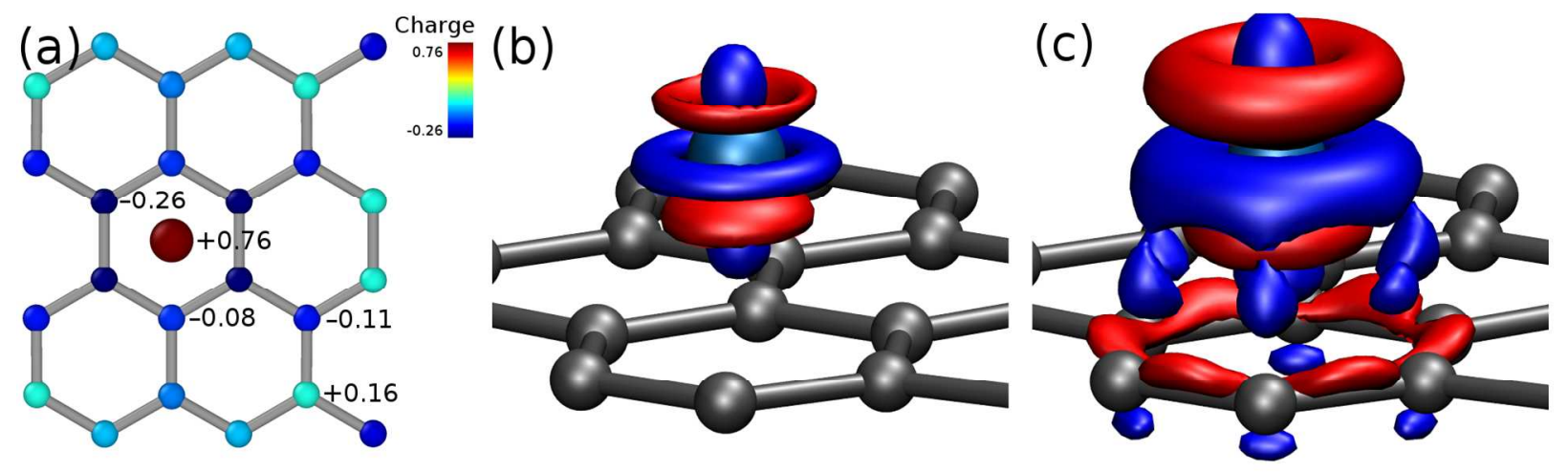

Figure 8. (a) Visualization of the Bader charges in the adatom system. (b), (c) Charge density difference plot of the Re atom on graphene, corresponding to the isovalues $0.03 \mathrm{e} / \mathrm{bohr}^{3}$ (b) and $0.01 \mathrm{e} / \mathrm{bohr}^{3}$ (c). Red and blue indicate depletion and accumulation of charge, respectively.

Clusters geometries were then selected for study using DFT from possible 3D structures observed in STEM images. All structures (after MD optimization) are shown in Table 2, along with the adsorption and interaction energies calculated from DFT. The normalized adsorption energy per atom and the shortest Re-surface perpendicular distance are also shown for comparison. Structures $\mathbf{3}$ and $\mathbf{5}$ were tested in different initial orientations. The tendency of decreasing normalized adsorption energy with increasing cluster size is not observed in this system. The reason lies in the fact that, in addition to the size effect, spin also shifts the energy considerably. While in all cases the total magnetic moment is lower for the Re adsorbed than in the vacuum, the quantitative change in magnetic moment is determined by the adsorption 
geometry. This leads in some cases to a strong distortion from the cluster in vacuum in order to create bonds with the surface, reducing the magnetic moment. This distortion represents a big positive term in the adsorption energy which in some cases, like structures 5 and 8 , exceeds the stabilization caused by the interaction. A detailed analysis about the effect of spin can be found in the Supporting Information.

With exception of the adatom, all clusters are adsorbed at a similar distance from the surface, between 1.9 and $2.2 \AA$. However the distance is not directly correlated with the normalized adsorption energy as is usually observed because of the spin effect as explained above.

\begin{tabular}{|c|c|c|c|c|c|c|}
\hline & Label & $\begin{array}{c}\mathrm{N}^{\circ} \text { of } \mathrm{Re} \\
\text { atoms }\end{array}$ & $E_{\text {int }}(\mathrm{eV})$ & $E_{a d s}(\mathrm{eV})$ & $\begin{array}{c}E_{a d s} / \text { atom } \\
(\mathrm{eV})\end{array}$ & $\begin{array}{c}D(\mathrm{Re}- \\
\text { surface }) / \AA\end{array}$ \\
\hline \multirow[t]{9}{*}{ Adatom } & 1 & 1 & -0.248 & -0.136 & -0.136 & 1.612 \\
\hline & 2 & 4 & -1.394 & -1.126 & -0.281 & 1.946 \\
\hline & $3 t$ & & -1.210 & -1.088 & -0.181 & 2.064 \\
\hline & $3 e$ & 6 & -1.349 & -1.013 & -0.169 & 2.220 \\
\hline & $3 s$ & & -1.769 & -1.258 & -0.210 & 2.073 \\
\hline & 4 & 8 & -2.332 & -1.583 & -0.198 & 1.829 \\
\hline & $5 t$ & & -2.581 & 0.088 & 0.011 & 1.922 \\
\hline & $5 e$ & 8 & -2.574 & 0.090 & 0.011 & 1.943 \\
\hline & 6 & 8 & -1.639 & -1.216 & -0.152 & 1.888 \\
\hline
\end{tabular}




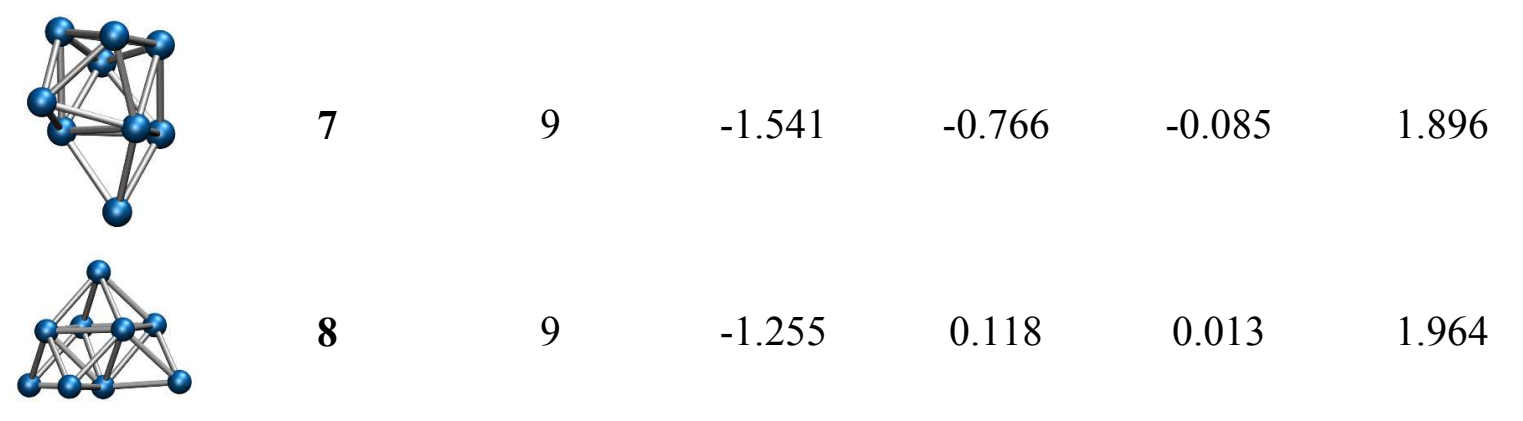

Table 2. Structures that were optimized on graphene, their labels, interaction and adsorption energy on graphene, adsorption energy normalized per Re atom and closest Re-surface distance.

In Figure 9 snapshots of all structures after relation are shown. As a general observation, it can be inferred that the structures distort to maximize the interaction of Re atoms with hollow sites. That is why, for instance, the tetrahedron (2) increases one Re-Re distance with respect to vacuum (which is compensated by the interaction with the surface), while one Re atom is distanced further from the surface.

Snapshots of different orientations 3t, 3e and 3s of the octahedron are shown in Figure 9 (b), (c) and (d). Two of these orientations were seen in STEM images (interacting with the vertex and the edge of the octahedron), while the third one (side of the octahedron in contact with the surface) is also a likely configuration. The octahedron is clearly the most stable structure than can be adsorbed, as it is concluded from the small distortion and high adsorption energy. In fact, structures 3 through 8 can also be seen as an octahedral seed, with Re atoms three-coordinated in sides of the octahedron. This explains their stability when adsorbed.

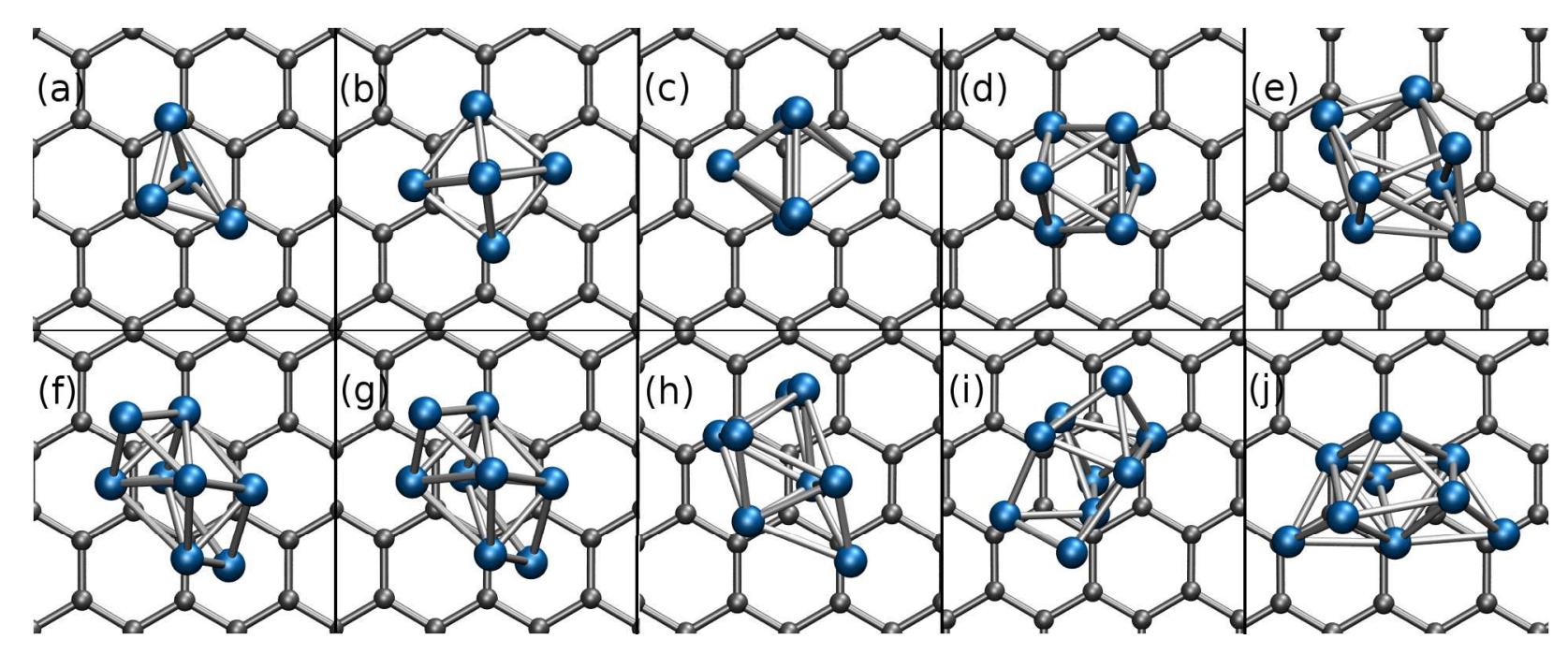


Figure 9. Top view of structures 2 (a), 3t (b), 3e (c), 3s (d), 4 (e), 5t (f), 5e (g), 6 (h), 7 (i) and 8 (j) before after relaxation on graphene.

The experimental Re-Re interatomic distance and interplanar distance were compared with the calculated ones for the tetrahedral (2) and octahedral (3e) structures. As in can be seen in Figure 5 , the measured interatomic distance in the tetrahedral structure is $0.25 \mathrm{~nm}$, while the average calculated Re-Re distance is $0.23 \mathrm{~nm}$. In the octahedral case, the experimental Re-Re distance $(0.25-0.26 \mathrm{~nm})$ is comparable to the calculated one $(0.24 \mathrm{~nm})$, and the measured interplanar distance $(0.21 \mathrm{~nm})$ is similar to the calculated distance $(0.20 \mathrm{~nm})$. The small differences can be attributed to the well-known overestimation of interatomic distances in the generalized gradient approximation. (19)

Finally, the Bader charges of the atoms of structures 2, 3t and 4 were calculated and are shown in Figure 10. The cluster polarization is noticeable in the three clusters depicted, the interacting atom/s having high positive charge, while the atoms at the top of the clusters bear small negative charges. The graphene surface has periodically alternated positive and negative charges with prominence of negative charge that compensates for the overall positive charge of the adsorbed clusters.

The net positive charge of the clusters has an important effect in catalysis. The catalytic effect depends on the reactivity, which is increased when the $d$-band approaches the Fermi level (20). As it is seen in the density of states (DOS) in the Supporting Information, the $d$-states of the rhenium hybridize with the states of graphene, generating $d$-states close (spin up) and above (spin down) the Fermi level which are unoccupied. Therefore, a higher catalytic effect can be expected in Re clusters when adsorbed on graphene than in isolated ones. 

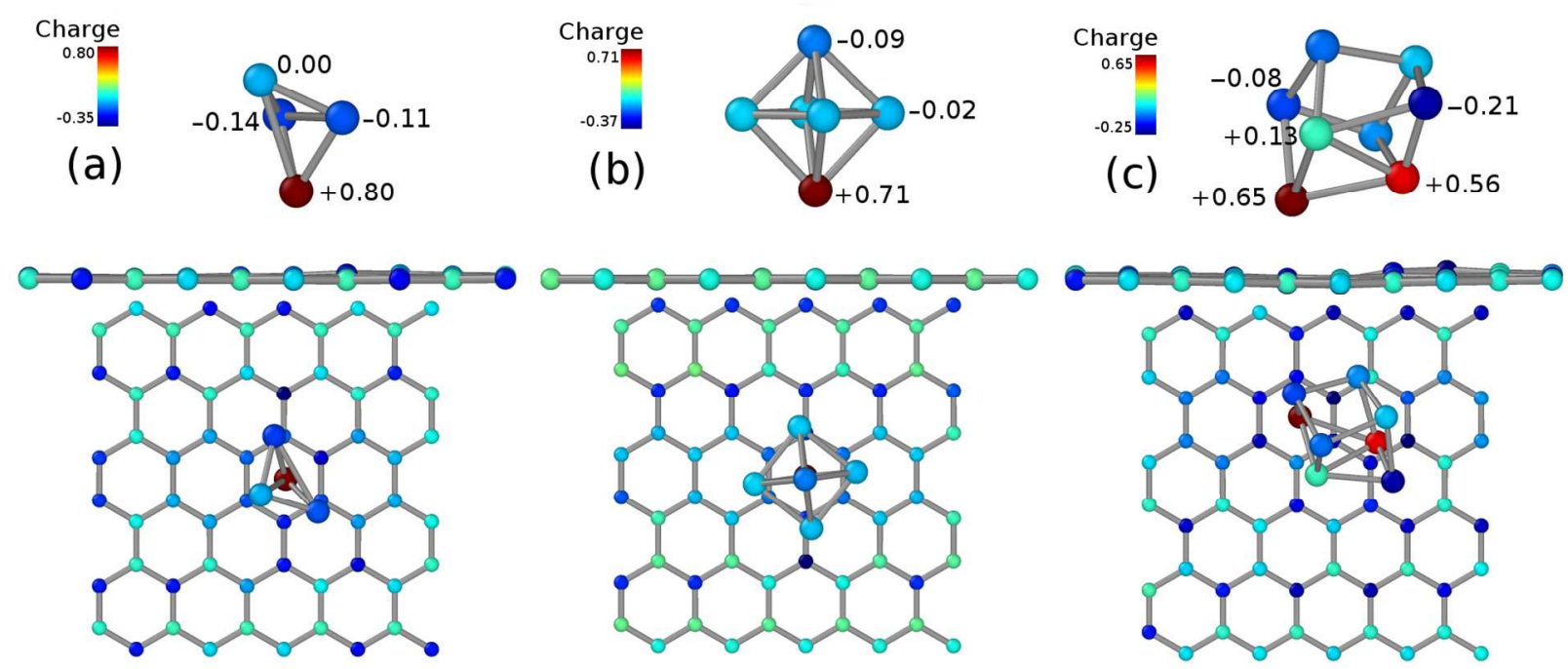

Figure 10. Side (top figure) and top (bottom figure) visualization of the Bader charges in structures (a) 2, (b) $3 t$ and (c) 4 on graphene. Note that the scale is normalized according to the charge range in each system.

\section{Conclusions}

Synthesis of rhenium clusters by a modified two-phase Brust method led us to obtain very small and low-atomicity clusters as well single Re atoms. Through a HAADF-STEM analysis and the intensities method we were able to distinguish the clusters that are formed by 2 or up to 12 atoms and determine their full atomistic configurations. The structures formed were very definite and simple, based on two 'seed' structure (octahedron and tetrahedron).

Density functional calculations were performed to explain the stability of the structures observed with STEM. An excellent agreement was found when compared the theoretical prediction with the experimental images. In particular the DFT calculations show that the octahedron is clearly the most stable structure than can be adsorbed.

An additional finding is reported, which open the possibility to develop ultra-small catalyst based on Re clusters adsorbed on graphene, i.e. higher catalytic effect can be expected in Re clusters when adsorbed on graphene.

\section{Acknowledgments}

The authors are indebted for support to the following: Welch Foundation Agency (project AX1615), The National Science Foundation (NSF) (PREM grant number DMR-0934218, NSF 
Grant 1103730) and NIH RCMI Nanotechnology and Human Health Core (Grant 5G12RR013646-12) at The University of Texas at San Antonio (UTSA). The group of Argentina wishes to thanks the CONICET, Universidad Nacional de Córdoba, ANPCyT Program BID (PICT 2010-1233) for financial support. The authors acknowledge the Texas Advanced Computing Center (TACC).

\section{References}

1. S. Chrétien, S. K. Buratto, H. Metiu, Current Opinion in Solid State and Materials Science 2007, 11, 62-75

2. X.-F. Yang, A. Wang, B. Qiao, J. Li, J. Liu, T. Zhang, Acc. Chem. Res. 2013, 46, 1740-1748

3. T. Epicier, K. Sato, F. Tournus and T. Konno, J. Nanopart. Res. 2012, 14, 1106

4. P. M. Voyles, D. A. Muller, J. L. Grazul, P. H. Citrin, and J. L. Gossmann, Nature 2012, 416 $823-829$

5. J. M. LeBeau, S D Findlay, L J Allen and S Stemmer, Phys. Rev. Lett. 2008, 100, 206101

6. J M LeBeau, S D Findlay, L J Allen and S Stemmer, Nano Letters 2010, 10, 4405-4408

7. A. Corma, P. Concepcion, M. Boronat, M. J. Sabater, J. Navas, M. J. Yacaman, E. Larios, A. Posadas, M. A. Lopez-Quintela, D. Buceta, E. Mendoza, G. Guilera, A. Mayoral, Nature Chem. 2013, 5, 775-781

8. B. Qiao, A. Wang, X. Yang, L. F. Allard, Z. Jiang, Y. Cui, J. Liu, J. Li, T. Zhang, Nature Chemistry 2011, 3, 634-641

9. Z. Y. Li, N. P. Young, M. Di Vece, S. Palomba, R. E. Palmer, A. L. Bleloch, B. C. Curley, R. L. Johnston, J. Jiang and J. Yuan, Nature 2008, 451 46-49

10. M. Brust, M. Walker, D. Bethell, D. J. Schiffrin and R. Whyman, J. Chem. Soc., Chem. Commun. 1994, 801-802

11. S. Plimpton, J. Comp Phys 1995 117, 1-19

12. G. Wang, M. A. Van Hove, P. N. Ross, and M. I. Baskes, J. Chem Phys. 2014, 121, 5410-5422

13. J. P. Perdew, J. A. Chevary, S. H. Vosko, K. A. Jackson, M. R. Pederson, D. J. Singh, and C. Fiolhais, Phys. Rev. B 1992. 46, 6671

14. P Giannozzi, S. Baroni, N. Bonini, M. Calandra, R. Car, C. Cavazzoni, D. Ceresoli, et al. J. Phys: Condens. Matter 2009, 21, 395502

15. W. Tang, E. Sanville, and G. Henkelman, J. Phys.: Condens. Matter 2009, 21, 084204. 
16. M. Zhou, A. Zhang, Z. Dai, Y. Ping Feng, and C. Zhang, J. Phys. Chem. C 2010, 114, 1654116546

17. C. Kittel, Introduction to Solid State Physics, 8th edition. Hoboken, NJ: John Wiley \& Sons, Inc. 2005

18. K. T. Chan, J. B. Neaton and L. M. Cohen, Phys. Rev. B 2008, 77, 235430

19. J. P. Perdew, K. Burke, M. Ernzerhof, Phys. Rev. Lett 1996, 77, 3875

20. E. Santos and W. Schmickler, ChemPhysChem 2006, 7, 2282-2285 